\title{
La filosofía del lenguaje: su naturaleza y su contexto
}

\author{
FRANCISCO RODRÍGUEZ-CONSUEGRA \\ Departamento de Lógica y Filosofía de la Ciencia \\ Universidad de Valencia \\ francisco.rodriguez@uv.es
}

\begin{abstract}
Resumen: El artículo es un intento de determinar la naturaleza de la filosofía del lenguaje a través de las relaciones de esa disciplina con otras, tanto desde el punto de vista histórico como desde el sistemático. Se examina la forma en que la filosofía del lenguaje ha venido relacionándose de hecho con la lingüística, la lógica, la psicología y la propia filosofía, al tiempo que se hacen propuestas de clarificación de esas relaciones, en el sentido prescriptivo del término. De paso, se critican ciertas nociones que han venido oscureciendo el problema, como las de "lógica filosófica", "filosofía lingüística" y otras, para terminar apoyando el papel fundamental de la filosofía del lenguaje dentro de la filosofía analítica, en un sentido amplio.
\end{abstract}

Palabras clave: filosofía del lenguaje, lógica, lingüística, psicología

Determinar con cierta precisión lo que es, o debe ser, la filosofía del lenguaje es tarea que usualmente no se aborda, o se hace de manera muy sumaria, casi siempre en la confianza de que su carácter resulte más o menos determinado mediante la mera exhibición de unos cuantos temas que configuran su contenido de facto. Naturalmente, ello presenta el inconveniente de que no se sabe entonces en virtud de qué criterios se ha llevado a cabo la selección de esos temas, con lo que no se sabe tampoco si se han escogido de entre los que habitualmente ocupan a los filósofos del lenguaje, o si meramente se está tratando de proponer una especie de programa de trabajo. Con estas consideraciones he aludido ya al carácter metafilosófico del título, así como a sus connotaciones necesariamente descriptivas y/o prescriptivas. Veamos más acerca de ello. ${ }^{1}$

De entre los rasgos más convincentes que se han propuesto para determinar el carácter específico de la filosofía, particularmente en oposición a las ciencias, está aquel que consiste en decir (Danto 1968) que la filosofía contiene como problema interno el determinar su propia naturaleza. Tal problema inaugura precisamente lo que se entiende por metafilosofía, mientras que las ciencias se limitan a inquirir sobre su contenido específico, dejando para la filosofía el determinar su naturaleza, lo que constituye

\footnotetext{
${ }^{1}$ Agradezco a Juan José Acero las observaciones que me hizo llegar tras su amable lectura de una versión anterior de este intento de determinar la naturaleza de la filosofía del lenguaje. Gracias a ellas he podido introducir mejoras en varios lugares.
} 
para ellas un problema externo. Pues bien, no parece caber duda de que, al menos por los mismos motivos, las divisiones fundamentales de la filosofía - como la filosofía del lenguaje- deberían también abordar la misma tarea, sobre todo en el caso típico en el que tal pregunta se suele plantear, que es justamente aquel en el que se trata de determinar después un contenido temático. Así, qué es —o qué debe ser- la filosofía del lenguaje es —o debería ser- uno de los problemas internos fundamentales de la filosofía del lenguaje.

Sin embargo, el declararlo meramente así nos lleva inevitablemente al problema de la determinación del contenido restante de tal especialidad. ¿Podemos decir — como irónicamente sugirió Russell respecto a la filosofía en general- que la filosofía del lenguaje consiste en aquello de lo que tratan los libros de filosofía del lenguaje? ¿O deberíamos más bien aportar, aunque fuera estudiando tales contenidos, ciertos criterios que sirvieran para determinarlos? El dilema es ciertamente algo ocioso, pues sabido es que en filosofía ambas tareas suelen hallarse estrechamente ligadas. Así, difícilmente podríamos dilucidar el concepto general con independencia de lo que en el uso normal cae bajo él, o, a la inversa, clasificar una serie de temas como pertenecientes o no a su extensión sin manejar de hecho criterios efectivos de clasificación. Por consiguiente, no parece que sea posible decidir a priori qué porcentaje prescriptivo contiene - debe contenernuestra determinación metafilosófica de la naturaleza de la filosofía del lenguaje sin, eo ipso, servirnos en algún grado del estudio de las temáticas que usualmente pasan por pertenecerle.

Ahora bien, constituiría ciertamente una tarea prolija el examen de esas temáticas, con vistas a su inclusión o no en la extensión que deseamos caracterizar, con independencia de la consideración de otras especialidades, más o menos cercanas, por comparación con las cuales es precisamente más dificultosa aquella caracterización. Así, parece conveniente abreviar algo nuestra tarea reconduciéndola a una comparación con aquellas especialidades que tenga por objeto no sólo el señalar con cuáles de ellas no coincide la filosofía del lenguaje y por qué (Valdés 1991, introducción), sino también, sirviéndonos de las diferencias halladas —en parte descriptiva, en parte prescriptivamente-, configurar con alguna claridad el carácter que buscamos. Es obvio que ello nos compromete necesariamente con la complicación de dar por supuesto mucho con respecto a la naturaleza de esas otras especialidades cercanas, pero no parece que sin asumir esa confrontación podamos hacer mucho más que poner de manifiesto nuestras preferencias.

Pues bien, de entre las materias con las que más se entrecruza la temática de la filosofía del lenguaje están sin duda, al menos por tradición, la filosofía lingüística y su compañera de viaje, la filosofía analítica (presumiblemente basada en el método del análisis filosófico), por lo que la 
comparación debería quizá comenzar con ellas. Sin embargo, con objeto de facilitar la tarea me parece más aconsejable despejar algo el camino dedicándonos primero a dilucidar los posibles rasgos comunes con otras disciplinas que, por diversas razones, suelen considerarse muy relevantes al respecto, como la lingüística, la lógica y la psicología, sobre las que diré algo, precisamente en ese orden.

\section{Filosofía del lenguaje y lingüística}

\subsection{Lenguaje, lingüística y filosofía}

La diferencia obvia entre filosofía del lenguaje y lingüística parece a primera vista suficiente. Ambas toman como objeto de estudio el lenguaje, pero mientras que la primera lo hace desde un punto de vista y con unos métodos filosóficos, la segunda lo hace desde el punto de vista científico y por ello con los métodos científicos habituales. Con un poco más de precisión, podríamos añadir que a la filosofía le interesan los problemas filosóficos que plantea el lenguaje, mientras que la lingüística busca elaborar teorías -científicas- que den cuenta de él. Es fácil mostrar que en cuanto tratamos de precisar un poco más, comienzan los problemas de solapamiento. Me limitaré a ofrecer unas cuantas ilustraciones de esas dificultades.

Ante todo está la noción misma de lenguaje. Uno de los problemas propiamente científicos de la lingüística consiste en elaborar teorías que nos digan qué es el lenguaje, tanto desde la óptica puramente estructural —formal, lógica, matemática-, como apoyándose en ciencias o subdisciplinas —-semiología, psicolingüística, sociolingüística, antropología, etc.- , que ensanchen ese marco hasta hacer justicia a la complejidad que aquél presenta. Pero es obvio que en el transcurso de ese esfuerzo se plantean habitualmente muchos problemas que poseen tintes claramente filosóficos. Por ejemplo, el problema —-metodológico, ontológico— del carácter de las reglas - sintácticas - que determinan el dominio de un lenguaje, desde el punto de vista de si su determinación puede o no presentarse como una explicación de esa facultad; o el problema —epistemológico- de la dilucidación del tipo de conocimiento que supuestamente tenemos de tales reglas.

A la inversa, el estudio filosófico del lenguaje requerirá cierta dependencia respecto de la lingüística, para que esa ciencia aporte con cierto grado de precisión el fenómeno que se va a estudiar, mientras que tal aportación no se puede aceptar sin analizar críticamente los supuestos o implicaciones -metodológicos, ontológicos, epistemológicos- que quizá presente la lingüística misma. Y, lo que es aún peor, a menudo los filósofos tratan de dilucidar por sí mismos la noción de lenguaje basándose exclusivamente en las redes conceptuales que les aportan sus propios presupuestos, sean 
éstos de carácter puramente filosófico o dependan de otras ciencias (como sucede, en el caso de la semántica modelista, de la lógica y la teoría de conjuntos), sin excesivo respeto por la lingüística, que sería una ciencia "meramente" empírica.

Otra dificultad de solapamiento se refiere de forma mucho más concreta al contenido de ambas materias. No parece que la fonética y la fonología planteen muchos problemas filosóficos; pero detengámonos, por ejemplo, en la semántica. Es innegable que la semántica es una parte importante de la lingüística, que se ocupa de nociones como significado, referencia, sentido e interpretación. Sin duda, su forma de ocuparse de ellas está dirigida al estudio de lenguajes concretos y a la forma en que tales nociones se manifiestan en ellos; pero caben pocas dudas de que a menudo los lingüistas ambicionan mucho más y apuntan hacia lo que verdaderamente hay de común en ellas, lo cual llevaría a hablar de supuestas facultades humanas universales. Y ¿cómo negar las implicaciones filosóficas de tales pretensiones? Cabe plantear lo propio respecto de la pragmática, incluso de forma más clara. Aquí no es ya sólo que tanto en obras de lingüística (de lingüistas) como de filosofía del lenguaje (de filósofos) puedan hallarse temáticas (por ejemplo, fuerza ilocutiva, implicatura conversacional) y autores comunes (por ejemplo, Austin, Searle, Grice), sino que es casi imposible deslindar con precisión quirúrgica lo que de científico — lingüístico- o de filosófico hay en sus tratamientos.

\subsection{Lingüística filosófica y filosofía de la lingüística}

Problemas como éstos parecen haber llevado a algunos a acuñar la desafortunada expresión "lingüística filosófica" (Acero et al. 1982). Un primer problema con ella es que parece sugerir que existe una lingüística científica y otra de carácter "racional" que, al estilo quizá de la vieja física filosófica aristotélica, trataría de abordar ciertos problemas, esquivos al tratamiento habitual, mediante otro tipo de métodos y quizá de conocimiento. Claro que no es eso lo que de hecho sus proponentes tienen en mente; pero la expresión no deja de ser peligrosa por las razones señaladas, especialmente si nos percatamos de que, como veremos más adelante, algo parecido sucede en el caso de la lógica, la matemática y quizá también en el de la psicología.

Más concretamente, se ha utilizado la expresión mencionada para abarcar el análisis pragmático del lenguaje, o la pragmática a secas, pero creo que sería mucho mejor hablar simplemente de pragmática y reconocer que tanto la filosofía como la lingüística tienen cosas serias que decir en ese campo (o quizá en esa rama de la semiótica). O bien, dejar los problemas pragmáticos propiamente dichos para la lingüística y aislar las implicaciones filosóficas de tales problemas — sus vertientes epistemológicas u 
ontológicas - con objeto de pasarlas a la filosofía, naturalmente con independencia de que hayan sido precisamente filósofos quienes hayan hecho aportaciones capitales a su estudio. Otras veces (Otero 1989) se ha entendido por lingüística filosófica simplemente la concepción de la filosofía del lenguaje que no tiene una relación directa con la lingüística, es decir, que no depende de ella en grado alguno, constituyendo así una aproximación precientífica a aquella ciencia. En tal caso se ha propuesto también que habría otra concepción de la filosofía del lenguaje, seguramente más auténtica, que vendría a coincidir con la metodología o filosofía de la lingüística (y más en general de la psicología cognitiva), en el sentido en que se entiende habitualmente la filosofía de una ciencia particular cualquiera.

Semejante propuesta me parece peor todavía. Por un lado, llamar lingüística filosófica tanto a la semántica modelista (o "modélica", según traduce Otero) como a la concepción de Davidson, metiendo en el mismo saco y sin distingos a toda la base fregeana, tarskiana y quineana, parece una simple fórmula para salir del paso a tiro de manual. Por otro, restringir la filosofía de la lingüística al puro estudio de las implicaciones filosóficas de la gramática generativo-transformacional parece algo más que mero sectarismo, por cierto muy propio de los seguidores (¿fieles?) de Chomsky. Esto último puede verse también en la propuesta más elaborada de Katz, que, como es sabido, ha pasado de defender la identificación entre filosofía del lenguaje y filosofía de la lingüística a separarlas de forma tajante, dejando para la segunda el estudio de las teorías y la metodología de la lingüística, y para la primera el estudio de la estructura del conocimiento conceptual, que presumiblemente se logrará a través de la teoría empírica del lenguaje que en todo caso ha de aportar la lingüística (véanse Katz 1966 y 1971).

La evolución de Katz me parece muy positiva; también yo creo que filosofía del lenguaje y filosofía de la lingüística no pueden identificarse. El problema radica en que, tanto en su primera etapa como en la segunda, Katz presupone sencillamente que la filosofía del lenguaje así como la filosofía de la lingüística vendrían a ser, unidas o separadas, nada más que una especie de apéndice filosófico del modelo chomskyano. Ello constituye un problema, al menos porque: (i) no todos las cuestiones de la filosofía del lenguaje pueden apoyarse en la lingüística generativo-transformacional; (ii) la lingüística chomskyana no agota toda la lingüística; (iii) la separación propuesta, siendo aceptable, no clarifica las relaciones entre los referentes de ambas expresiones; de hecho ni siquiera determinan su extensión o campo de aplicación. Vayamos por partes.

Es obvio que existen temas interesantes de la filosofía del lenguaje que muy poco o nada tienen que ver con la lingüística generativa. Así, el tema de la referencia directa, el de las implicaciones filosóficas de las gramáticas de Montague, o el de la supuesta importancia de una teoría general del significado para abordar problemas filosóficos básicos (al estilo de Dum- 
mett). Claro está que siempre puede decirse que la aproximación concreta a tales problemas está precisamente en su recolocación dentro del ámbito de la lingüística generativa, mientras que si ello no es posible, entonces es que no se trata de temas o problemas de interés lingüístico genuino. Pero semejante reacción no pasaría de mera táctica dilatoria, al tiempo que estaría aceptando claramente que podrían existir problemas filosóficos genuinos que dependieran de otras líneas. Que la lingüística generativotransformacional no agota toda la lingüística es también evidente; no hay más que abrir textos de lingüística para comprobarlo. Y no me refiero sólo a temas pragmáticos, sino a muchos temas semánticos, o relacionados con la cultura, la psicología o la sociología. Por todo ello, el aceptar la separación entre filosofía del lenguaje y filosofía de la lingüística, aun reconociendo la vital importancia del modelo chomskyano para ambas, no nos debe comprometer con una dependencia demasiado estrecha de ese modelo en ninguno de los dos sentidos. Queda el tercer punto de los señalados.

La relación que me parece que existe $-\mathrm{o}$ al menos debe existir- entre filosofía del lenguaje y filosofía de la lingüística es la de inclusión propia: entre los problemas de la filosofía del lenguaje se hallan los de la filosofía de la lingüística. Así, aunque todos los problemas filosóficos de la lingüística son, en principio, problemas de filosofía del lenguaje, esta última es una materia más amplia en la que caben otras temáticas. No parece necesario mostrar lo primero (véase, no obstante, infra), así que basta probar lo segundo, para lo que es suficiente recordar los ejemplos ofrecidos en el párrafo anterior u otros similares. Ello no significa que la filosofía de la lingüística no sea relevante para la filosofía del lenguaje; lejos de ello, reconocer la relevancia de la lingüística es reconocer, a fortiori, la de su estudio filosófico (es decir, semántico, epistemológico y ontológico). De lo que se trata es simplemente de darse cuenta de que no toda filosofía del lenguaje es filosofía de la ciencia. Muchos de los problemas más genuinos de la filosofía del lenguaje son problemas conceptuales referidos al lenguaje mismo, o a nociones estrechamente relacionadas con él, como las de pensamiento, mente o acción, más bien que al estudio filosófico de la ciencia de la lingüística, sus teorías o sus métodos.

Insisto; no se trata de defender una aproximación "filosófica" independiente a los objetos mismos de la lingüística, ni tampoco — sólo— de señalar problemas no estrictamente lingüísticos en filosofía del lenguaje. Se trata simplemente de llamar la atención sobre la clara diferencia existente entre filosofía de cierto objeto o proceso y filosofía de la ciencia que estudia ese objeto o proceso (por más que los propios científicos ignoren a menudo esa diferencia). La filosofía de la lingüística no estudia los objetos de la lingüística como tales (eso ya lo hace la lingüística misma), sino sólo como construcciones teóricas, con el fin de dilucidar y evaluar sus virtudes teórico-metodológicas y sus implicaciones filosóficas. La filosofía del len- 
guaje es más amplia, pues se abre al lenguaje también desde el punto de vista del conocimiento precientífico, de la terminología corriente, de sus relaciones con otras nociones con las que lo hallamos emparentado y de otros problemas filosóficos no relevantes para la filosofía de la ciencia.

Naturalmente, debemos estar preparados, una vez rebasada esta primera aproximación conceptual, para encontrarnos con problemas más o menos especializados que, perteneciendo claramente a la filosofía de la lingüística, constituyan dudosamente problemas propios de la filosofía del lenguaje. En tal caso diremos que la filosofía del lenguaje debería hallar una lectura más puramente filosófica y general de tales problemas, tratando de rebasar el más estrecho marco de la ciencia de la lingüística. Está claro que para ello deberemos contar con categorías filosóficas propias, y eso requiere, a su vez, teorías propiamente filosóficas, pero eso suele ser lo habitual.

Resumiendo: la filosofía del lenguaje debe mirar atentamente la ciencia de la lingüística, pero no se confunde con la filosofía de la lingüística, sino que la incluye, mientras que deberíamos deshacernos de la expresión "lingüística filosófica” por desorientadora e inútil. Como ocurre con la matemática y la física, podremos también, al considerar ciertas partes o resultados de la lingüística, hablar de sus implicaciones filosóficas.

\section{Filosofía del lenguaje y lógica}

\subsection{Forma lógica, lenguaje y filosofía}

Históricamente, las relaciones entre filosofía y lógica han sido estrechas y variadas, ya desde Aristóteles. Sin embargo, las características particulares de la relación de la moderna filosofía del lenguaje con la lógica proceden de los inicios de la propia filosofía del lenguaje, es decir, de la época en que los creadores de la filosofía analítica dieron en: (i) crear lenguajes formales con objeto de formalizar las proposiciones de la matemática (Frege, Peano, Russell); (ii) proceder al análisis sistemático de las ideas implicadas en los fundamentos de la matemática, mediante la creencia de que el resultado de tal análisis es la obtención de la forma lógica auténtica subyacente en la forma gramatical engañosa (Frege y Russell, con el antecedente de Bradley desde fuera del campo matemático), y (iii) aplicar los métodos obtenidos en tal análisis a otros problemas filosóficos, extendiendo la creencia de que la búsqueda de formas lógicas es lo que debe caracterizar la filosofía, hasta llegar a los componentes últimos e indefinibles de las proposiciones filosóficamente interesantes (Moore, Russell, primer Wittgenstein).

Podemos ilustrar esos tres momentos, tan sólo artificialmente separados en pro de la claridad en la exposición, considerando brevemente su aparición en la propia obra de Russell, que puede considerarse el fundador 
supremo de la filosofía del lenguaje en el sentido moderno. La utilidad de los lenguajes formales se halla perfectamente disecada en la introducción a la primera edición de los Principia. Russell dice allí que el uso del simbolismo en que se decantan: (i) hace posible establecer la conexión con la necesidad de manejar ideas sumamente abstractas, para las que el lenguaje ordinario no aporta términos; (ii) proporciona los recursos de simplicidad requeridos para reproducir los procesos del razonamieto deductivo; (iii) supera las limitaciones de la imaginación a la hora de manejar conjuntos y series de ideas abstractas, que no se ven representadas por la forma gramatical; (iv) hace posible abarcar proposiciones muy complejas de un golpe, y (v) permite llevar a cabo el mayor análisis posible de las ideas y proposiciones involucradas, hasta presentarlas en su aspecto más puramente formal.

No hay que olvidar que Russell se está refiriendo aquí explícitamente al programa logicista ideal de formalización de la matemática, pero sin duda también está pensando en logros ya habidos, como la teoría de las descripciones, en los que el hallazgo de formas lógicas no sólo permitió la obtención de instrumentos necesarios para proseguir el trabajo de formalización de la matemática, sino también el desvelamiento de estructuras lingüísticas subyacentes que iluminaban todo un campo de implicaciones filosóficas hasta entonces oscuro. En ese punto preciso es donde nuestro segundo momento aflora, mostrando cómo el análisis lógico de ideas lograba despojarlas de aditamentos gramaticales confundentes, en este caso sobre los conceptos de nombre, referencia, descripción, identidad, existencia, conocimiento directo, etcétera.

La culminación de todo ello radicó en la extensión de tales métodos hasta caracterizar el tipo de filosofía que interesaba a Russell. De ahí que el segundo capítulo de su obra sobre el conocimiento del mundo externo (1914) se titulase "La lógica como esencia de la filosofía", y que Russell estuviese hasta tal punto convencido de ello que llegara a considerar tales procedimientos como lo que caracteriza "El método científico en filosofía". En esos trabajos aparece claramente la idea de que la utilización filosófica de la lógica consiste precisamente en el desvelamiento de las formas lógicas de las proposiciones filosóficamente relevantes, entendiéndose por forma simplemente la manera en que los diversos elementos constituyentes de la proposición están combinados entre sí, lo que naturalmente impide (a riesgo de regreso infinito) considerar esa forma como un constituyente más de la proposición. Así, el propósito del método científico en filosofía es hacer explícitas las formas auténticas que laten bajo las engañosas, como sucede, según Russell, con la tradicional forma sujeto-predicado. Y ello, naturalmente, no puede llevarse a efecto sin olvidar el problema básico de la naturaleza última de la proposición (o del juicio) que, como sabemos, se convirtió en el problema central del Tractatus (aunque ya lo fue para Brad- 
ley y en cierto sentido también para Moore; véase Rodríguez-Consuegra 2002, caps. 2 y 3 ).

En este sentido para Russell la filosofía no se puede separar de la lógica, pues sólo la lógica puede aportar la catalogación última del repertorio de formas lógicas posibles, y con ello de los diversos tipos de hechos y sus elementos constitutivos últimos, que es el resultado del análisis filosófico y constituye la esencia de lo que se conoce como atomismo lógico (expresión que se atribuye a veces erróneamente a Wittgenstein; véase RodríguezConsuegra 2002, caps. 5 y 6). Pues bien, como lo ilustra Russell con profusión en esas obras, la aplicación de tales métodos a problemas filosóficos diversos es lo que hace posible el verdadero éxito en filosofía. No hay más que recordar su análisis de nuestro conocimiento del mundo externo, en el que se aplican modelos puramente lógicos (matemáticos diríamos hoy) al lenguaje con el que describimos la realidad física, hasta descomponer sus proposiciones en elementos constitutivos genuinos, es decir, en átomos lógico-epistemológicos con los que, según Russell, estemos auténticamente familiarizados.

Este último momento, que como es obvio rebasa lo que puede entenderse por filosofía del lenguaje, tenía por fundamento, sin embargo, una de las dos partes en que, según Russell, se divide la lógica; en concreto, aquella parte inicial en la que distinguimos y catalogamos las formas lógicas, y a la que bautizó, de forma históricamente feliz a juzgar por el éxito de la expresión, como lógica filosófica (siendo la otra parte la puramente matemática en la que obtenemos consecuencias, es decir, demostramos teoremas). Así, puede decirse que para Russell la lógica filosófica es la esencia de la filosofía, en el sentido preciso en el que la esencia de la filosofía es la filosofía del lenguaje, dado que su cometido principal debe ser el análisis lógico de las proposiciones hasta desvelar su naturaleza última — sin olvidar las implicaciones del tema para con la filosofía de la mente, a través del análisis del juicio y el de las actitudes proposicionales (expresión ésta, por cierto, también debida a Russell).

Aquí tenemos ya el menú principal de lo que ha venido siendo, durante muchas décadas, la aproximación típica a la filosofía del lenguaje $-\mathrm{y}$ a gran parte de la filosofía de la mente- característica de la tradición analítica. No tenemos más que añadir el toque final mediante el cual Russell estableció el enlace con Wittgenstein y el positivismo lógico. El primer paso se concretó en la identificación de las formas lógicas con las constantes lógicas (Russell 1919). Como es sabido, Russell y Wittgenstein no se pusieron de acuerdo en su interpretación filosófica, pero lo que debemos retener ahora es que Russell estableció el espacio filosófico relevante al sostener que lo auténticamente importante para el problema de la naturaleza de la lógica (de la filosofía de la lógica, diríamos hoy) es la naturaleza de sus constantes (o formas), al tiempo que hábilmente insertaba ese pro- 
blema en el marco general de su logicismo, al sostener la existencia de un continuo caracterizado por un comienzo filosófico (lógica filosófica), un tránsito lógico (lógica matemática) y un final matemático (aritmética, análisis y geometría).

El segundo paso dio lugar, tras unos primeros intentos de resistencia, a una rendición completa de Russell al punto de vista de Carnap, según el cual, aunque el objeto de la filosofía debe seguir siendo el análisis lógico, tal análisis no deja lugar para una consideración platónica de las constantes lógicas, sino que éstas deben desaparecer en el marco de un tratamiento convencionalista de la lógica y de una reducción lingüístico-sintáctica de las proposicones matemáticas (Russell 1951). Con ello, el programa de la "filosofía del lenguaje ideal" lograba mantenerse tras el problema de las paradojas y los ataques de Wittgenstein.

\subsection{Lógica filosófica y filosofía de la lógica}

El problema que plantea el pequeño estudio de los orígenes de nuestra disciplina del apartado anterior es que, con él en la mano, aunque entendemos mucho mejor el suelo que pisamos, nos surgen grandes dudas acerca de la posibilidad o conveniencia de seguir confiando en las etiquetas tradicionales que presupone el uso de la mayoría de las expresiones consagradas por la costumbre. Ello se concreta en la necesidad presente de clarificar las relaciones que existen —o deben existir — entre la filosofía del lenguaje, la lógica filosófica y su pariente cercano, la filosofía de la lógica. Planteado de forma más clara: (i) ¿podemos continuar manteniendo que la lógica filosófica busca las formas lógicas genuinas y es, en ese sentido, la esencia de la filosofía del lenguaje? Sólo a la luz de nuestra respuesta a esta pregunta podremos pasar a la siguiente: (ii) ¿qué relaciones debe tener la filosofía del lenguaje con la lógica filosófica y con la filosofía de la lógica?

La respuesta a la primera pregunta no puede ser más que negativa. Toda la tradición, digamos clásica, que siguió a Russell en la creencia de que el lenguaje natural ha de ser reducido a sus formas lógicas lo ha hecho con la vista puesta en lo que Quine llamó "la regimentación del lenguaje"; es decir, en la aportación de un modelo reductivo según el cual sólo es recuperable lo que encaja con el modelo aportado por la lógica de primer orden, y si hay estructuras lingüísticas -intensiones- que no encajan, peor para ellas. Sin embargo, para esa tradición misma, al igual que son perfectamente admisibles distintas reducciones conjuntistas de los números, con tal de que cada una cumpla las propiedades aritméticas que esperamos de ellos (véanse Rodríguez-Consuegra 1991 y 1992), así también deberían admitirse distintas formas lógicas para las proposiciones del lenguaje natural, con tal que todas cumplan las propiedades que esperamos de ellas, una vez 
inmersas en el sistema formal dado, de cara al conjunto total de la ciencia. En ese sentido no parece que pueda hablarse de formas lógicas genuinas como una especie de correlatos únicos a las enunciados lingüísticos, máxime cuando, incluso dentro de la esfera de la traducción misma, aparece enseguida el fenómeno de la indeterminación y la relatividad.

En cuanto a la línea de Davidson, heredera de Quine, también parece que la respuesta sería negativa. El sentido de las formas lógicas de Davidson es aproximadamente el mismo que el de las de Russell y Quine, sólo que complementado por el aditamento tarskiano de una teoría de la verdad en el sentido de una teoría del significado. Así, cualesquiera formas lógicas canónicas que se pongan en correspondencia con los enunciados del lenguaje natural, y que permitan la introducción de una semántica, son aceptables, puesto que las condiciones de verdad que establecen dan el significado auténtico del fragmento del lenguaje de partida. Pero el fenómeno de la indeterminación continúa amenazando la construcción completa, que en consecuencia no es menos holista por ello. En última instancia, la única forma de superar esa indeterminación debería ser la conexión de los enunciados originales con sus formas lógicas, partiendo de una gramática construida de tal modo que tuviera "realidad psicológica" (Lycan 1984), con lo que estaríamos hablando ya de la constitución misma del cerebro. Quizá cabría hablar entonces de formas genuinas, pero todo el asunto se escaparía de las manos del lógico y el adjetivo "lógicas" dejaría de tener utilidad. Seguiríamos, pues, respondiendo negativamente a nuestra pregunta.

En el caso de la semántica modelista, el problema se hace mucho más complejo, pero queda aparentemete resuelto señalando que sólo muy dudosamente cabe hablar aquí de algo parecido a lo que habitualmente se entiende como forma lógica (Gamut 1991), aunque desde luego no parece tampoco que pueda entonces hablarse de unicidad. En todo caso, los potentes instrumentos matemáticos manejados por la semántica modelista plantean incluso un problema de carácter inverso, pues podría incluso decirse, a la vista de sus construcciones, que de lo que se trata es de modelos matemáticos que ponen de manifiesto la naturaleza matemática del lenguaje. Como vio muy bien Gödel en algunos de sus manuscritos inéditos (Gödel 1994), si ello fuera así, la concepción neopositivista según la cual la lógica y la matemática son de carácter lingüístico estaría profundamente equivocada, y la verdad caería del lado de la tesis opuesta: el lenguaje mismo sería imposible sin matemática.

En el caso de la línea chomskyana se ha hablado también de formas lógicas, relacionadas de cierta manera con las estructuras sintácticas puestas de manifiesto por el análisis aportado por el modelo generativo-transformacional. Sin embargo, en la medida en que la decantación de tales formas lógicas presupone, para los seguidores de esa línea, la existencia de correlatos mentales, e incluso cerebrales, que les otorgan su realidad 
psicológica, entonces, aunque cabe hablar de autenticidad, de nuevo el adjetivo "lógicas" pierde su sentido. Naturalmente, fue por no afrontar peligros similares que el viejo Russell, que ya en su juventud había podido huir del psicologismo (en buena medida gracias al objetivismo lógico del propio Bradley), negó una y otra vez realidad mental subyacente alguna en las formas lógicas, que debían ser totalmente generales y a priori, en un sentido objetivista no kantiano, con objeto de marginar la vieja concepción de la lógica, según la cual ésta se limita a recoger las "leyes del pensamiento", que serían entonces subjetivas.

En pocas palabras: en ninguna de las líneas serias de trabajo conforme a las cuales puede todavía hablarse de formas lógicas cabe sostener, aunque sea por razones diversas, que tales formas sean únicas, en el sentido de la vieja creencia de Russell, Moore y Wittgenstein (y quizá de Frege, aunque con más dudas), según la cual las proposiciones sólo tienen un análisis lógico correcto en sus componentes últimos indefinibles. Falta por saber, antes de pasar a nuestra segunda pregunta, si tiene sentido para esas líneas de trabajo considerar que existe una lógica filosófica, como esencia de la filosofía del lenguaje, destinada a descubrir un repertorio de tales formas.

De nuevo, la respuesta ha de ser negativa, a pesar de ciertos lastres tradicionales (Sainsbury 1991). Ni Quine ni Davidson, que yo sepa, dan pie para hablar de una lógica filosófica tal, como suerte de fundamento de la lógica matemática, que nos permita la consecución de aquel repertorio. Así, sería la lógica matemática a secas, complementada con la semántica conjuntista, la encargada de aportarnos instrumentos para formalizar el lenguaje de manera satisfactoria. Con lo cual parece que cualquier evaluación de la selección de formas que se van a emplear tendría que estar en función de los fines que persiguiéramos en cada caso. Ello no quiere decir que no pueda discutirse si tal o cual formalización es mejor que otra; pero sí que hemos de especificar antes lo que pretendemos con ello, e incluso que somos nosotros los que introducimos la formalización más que descubrirla, como a veces parece que pretendía Russell. Por tanto, al negar carta de naturaleza a la lógica filosófica, en ese sentido preciso que estamos manejando ahora, no es posible convertirla en esencia de la filosofía del lenguaje.

Otra cosa es saber si esa esencia puede hallarse, de todas formas, en las respectivas ideas de formalización de Quine o Davidson. En lo que respecta a Quine, en ningún caso su propuesta de regimentación parece agotar aquella esencia. Es cierto que el modelo de formalización que ha propuesto constituye su instrumento favorito para atacar la exuberancia ontológica de las escuelas que ve como enemigas, muy en particular la de todo lo que huela a intensionalismo. Pero no debe olvidarse que para el viejo maestro de Harvard, los problemas de las implicaciones ontológicas de los lenguajes formales son también parte esencial de la filosofía del 
lenguaje, así como la indeterminación de la referencia y la traducción. Sin embargo, todos ellos pueden afrontarse con independencia del tema de las formas lógicas. En el caso de Davidson, la respuesta es menos clara, pues es indudable que su teoría de la verdad como teoría del significado es la esencia de su filosofía del lenguaje, así como que esa teoría de la verdad entra en la línea de lo que venimos llamando "formas lógicas". Pero ya hemos señalado que existen serias dificultades para insertar su concepción de las formas lógicas en el modelo clásico, principalmente por aproximarse a una teoría de la mente destinada a superar el problema de la indeterminación.

Pasemos ya brevemente a la segunda pregunta: ¿qué relaciones debe tener la filosofía del lenguaje con la lógica filosófica y con la filosofía de la lógica? Vistas las dificultades de abordar el tema de la lógica filosófica en la tradición de las formas lógicas, parece que lo más sensato sería prescindir de semejante expresión, que inevitablemente provoca la creencia en que la lógica se divide en dos partes: matemática y filosófica, y puede llevar al convencimiento de que existe un conocimiento científico de carácter filosófico (como veíamos en el caso de la rechazable "lingüística filosófica"). El problema radica en que existe una tradición bien establecida según la cual eso es precisamente así. No hay más que pensar en los títulos de dos célebres manuales canónicos, Barwise 1977 y Gabbay y Guenthner 1983-1989, para darse cuenta de ello. No es probable, por tanto, que una propuesta en ese sentido prospere. Ahora bien, tampoco me parece que el uso contemporáneo de la expresión sea muy claro, ni que, en consecuencia, coadyuve en lo más mínimo a establecer una relación precisa con la filosofía del lenguaje. Veámoslo.

En general, los tres primeros tomos del segundo manual citado son exposiciones de lógica, más o menos introductorias, pero técnicas, en el orden esperado: lógica clásica (primer orden, órdenes superiores, teoría de tipos), extensiones de la lógica clásica (lógicas intensionales) y alternativas a la lógica clásica (lógicas intuicionista, multivalente, multivariada). Ante todo está el hecho de que la lógica clásica esté también incluida, con lo que parecería que toda lógica es filosófica, aunque sin duda ello se hizo para dar una introducción clásica a los otros volúmenes. Podría quizá pensarse que lo que hace filosóficas a ciertas partes de la lógica es que por motivos históricos hayan estado asociadas a ciertos problemas filosóficos de múltiple origen, y desde luego no especialmente lingüístico, o quizá porque se presten más a extraer implicaciones filosóficas. Pero, aún así, el nexo de esa lógica "filosófica" con la filosofía del lenguaje no resulta transparente.

Es cierto que algunos de los temas recogidos, como la lógica intuicionista o la lógica modal, han dado paso a tendencias interesantes en la filosofía del lenguaje (Dummett, Kripke y la semántica modelista, respectivamente), pero ello no parece justificar el nexo, que no es en ningún caso general. 
Quizá, en última instancia, ese nexo radique en que, en la mayor parte de los casos, las ramas de lógica cubiertas han hecho posible la formalización de argumentos que no "cabían" en la lógica clásica de primer orden. Pero si ello fuera así, entonces estaríamos volviendo de manera subrepticia al enfoque de las formas lógicas, y ya hemos visto con algún detalle los problemas a los que ello conduce. Concluyo, por tanto, que sólo oscuras razones históricas de respeto a una tradición llevan a mantener la expresión "lógica filosófica".

El problema de la relación con la filosofía del lenguaje se ve agravado cuando observamos que el cuarto volumen de Gabbay y Guenthner se dedica precisamente a temas de filosofía del lenguaje. Con ello, sus compiladores parecen proponer que tal especialidad, o al menos una parte de ella, pase a estar incluida en la lógica filosófica, sea esto lo que fuere. La supuesta justificación podría radicar en que, en tales temas (el papel de los cuantificadores en la formalización; la presuposición; las descripciones y la referencia), el uso de técnicas lógicas es muy importante. Y, puesto que obviamente se trata de temas filosóficos, no hay más que unir los dos componentes - lógica y filosofía- y ya tenemos la lógica filosófica resultante.

Pero por razones similares podríamos haber añadido un quinto tomo a la serie en el que se tocaran temas de física, o de matemática, en los que el uso de técnicas lógicas fuese también importante, sin que por ello a nadie se le ocurriera hablar de lógica filosófica. Concluyo, de nuevo, que sólo razones históricas, unidas a razones corporativas y de estructura de los departamentos universitarios, permiten mantener el nexo tradicional entre esa oscura lógica filosófica y la filosofía del lenguaje. En cuanto a la lógica, ahora a secas, qué duda cabe de su gran papel en la filosofía del lenguaje, fundamentalmente a través de la creación de lenguajes formales y su aplicación a problemas de filosofía del lenguaje. Pero ello no exige, ni aconseja, hablar de lógica filosófica, puesto que aquellas aplicaciones son también posibles a partir de áreas de la lógica que nadie sostiene que sean filosóficas en el mismo sentido (teoría de modelos, teoría de conjuntos). Resumiendo: la lógica tiene importantes aplicaciones e implicaciones filosóficas en filosofía del lenguaje, pero eso no hace — no debería hacer"filosófica" a la lógica.

Queda sólo preguntarse por la relación entre filosofía del lenguaje y filosofía de la lógica. Aquí podemos ser mucho más breves, pues no se trata ya de ningún problema de solapamiento de la filosofía del lenguaje con otra disciplina no propiamente filosófica, como la lógica o la lingüística. Propongo resolver el problema de forma empírica. Tómense un par de textos de filosofía de la lógica de calidad y hágase la siguiente pregunta: ¿̇on relevantes consideraciones acerca del lenguaje natural y sus problemas filosóficos para dilucidar los problemas propios de la filosofía de la lógica? Veremos en seguida que, en la inmensa mayoría de los temas de nuestros dos libros, 
la respuesta no puede ser más que positiva. Así, los temas de la validez, la verdad lógica, la naturaleza de la lógica (de sus "objetos"), la de sus cuantificadores, los portadores de verdad, las teorías de la verdad, las paradojas, etc., etc., todos ellos reclaman perentoriamente el auxilio de aquellas consideraciones. Concluyo que existe un estrecho nexo entre filosofía del lenguaje y filosofía de la lógica. Ahora bien, ¿cuál es la naturaleza de ese nexo?

En parte ya lo hemos visto. La filosofía de la lógica necesita recurrir a la filosofía del lenguaje para abordar con éxito sus problemas. A la inversa, el problema es más complejo. No cabe duda de que el filósofo del lenguaje usa de hecho ideas extraídas del campo de la filosofía de la lógica con provecho; pero me parece que sólo de manera secundaria, y ello en temas bastante específicos (pienso sobre todo en Dummett, Davidson y Kripke). Me parece que lo que debería decirse es, simplemente, que hay filósofos del lenguaje que se inspiran en ciertos problemas de filosofía de la lógica para elaborar sus teorías, pero eso no parece reclamar la necesidad sistemática de hacerlo. En todo caso, el tema es demasiado oscuro como para decir cosas precisas con mucho sentido. En el fondo, todo parece resolverse en un sentido corporativo: hay filósofos que trabajan en ambos campos y otros que no; pues bien, es probable que los primeros hallen más fructífera la interacción que los segundos.

El nexo más importante es paralelo al que hemos hallado al estudiar las relaciones entre la filosofía del lenguaje y la filosofía de la lingüística: la aspiración generalizadora (categorizadora) de la filosofía del lenguaje hacia (o desde) la construcción de una filosofía superior (véase más adelante la sección 4). Ahí topamos con la inevitable dependencia de alguna teoría filosófica, o incluso claramente metafísica, que trate de arrojar luz sobre problemas filosóficos específicamente relacionados con el lenguaje, como sucede, digamos, con la posibilidad de construir auténticas teorías del significado. Así, por ejemplo, según manejemos una metafísica u otra, así entenderemos la causalidad (o incluso la idea misma de sustancia), y así también entenderemos el papel de las relaciones causales en posibles teorías del significado, sobre todo si éstas caen en el ámbito de algún programa de naturalización del significado, como empieza a ser habitual en nuestros días (véanse, por ejemplo, obras como Millikan 1984 y Schiffer 1987). Pero nada de ello nos forzará a confundir la filosofía del lenguaje con la filosofía de la lingüística o con la filosofía de la lógica.

\section{Filosofía del lenguaje y psicología}

3 . 1. Lenguaje, mente, y filosofía

Las nociones de lenguaje y mente cuadran bastante bien entre sí, sobre todo en la medida en que ambas proceden de etapas casi precientíficas 
de nuestra historia intelectual. Así, no es extraño que exista otra noción, la de pensamiento, que haya obrado durante mucho tiempo como campo de batalla donde se dirimen las disputas sobre sus relaciones, casi siempre cuando lo que se discute es - o era- si el lenguaje es mera expresión de un pensamiento preexistente, por tanto no lingüístico, o si por el contrario lenguaje y pensamiento vienen a coincidir en lo fundamental, por ser el pensamiento ya de carácter esencialmente lingüístico. En la medida en que pueda establecerse un continuo de posiciones en torno a estos dos polos, obtendremos fácilmente diversas concepciones de la mente.

La diferencia relevante entre ellas será, precisamente, que tendremos tanto más acceso a la naturaleza de la mente desde el estudio del lenguaje cuanto más lingüísticas creamos que son sus operaciones y contenido. En consecuencia, es obvio que la concepción del lenguaje que sostengamos será extraordinariamente relevante para nuestra concepción de la mente. Así, filosofía del lenguaje y psicología han estado, y pueden continuar estando, estrechamente relacionadas. Curiosamente, en una época como la nuestra, en la que algunos filósofos de éxito han llegado a dudar de que el término "lenguaje" posea referencia, y algunos psicólogos y filósofos han dirigido fuertes críticas contra la noción de mente, todavía existen disciplinas en buen estado de salud dedicadas a explorar ambas nociones y sus relaciones mutuas.

Obviamente, la posición más favorable a unas relaciones fructíferas entre el estudio de la mente y el del lenguaje es la que defiende la identidad entre lenguaje y pensamiento. Sin embargo, un problema especialmente difícil en torno a esa identidad es que puede defenderse desde posiciones radicalmente antagónicas. Por ejemplo, puede argumentarse que lenguaje y pensamiento coinciden porque existe un lenguaje del pensamiento de carácter innato, del cual los diferentes lenguajes naturales que aprendemos no serían más que una especie de transcripción, basada en posibilidades representacionales preexistentes (Fodor 1975). Desde esa óptica, el estudio de los "universales lingüísticos" arrojaría sin duda mucha luz sobre la naturaleza de aquel lenguaje representacional, y por tanto sobre la naturaleza del pensamiento, la mente y la cognición (sean éstas lo que fueren). Puede argüirse, en cambio, que esa coincidencia entre lenguaje y pensamiento tiene lugar tan sólo porque interiorizamos las estructuras lingüísticas que, como formas de comportamiento, utilizamos para comenzar a insertarnos en una red social que las utiliza en ese sentido. Con ello, un posible "lenguaje interior", lejos de constituir una parte originaria de la mente, no vendría más que a estar por unas relaciones perfectamente contingentes y variables de una cultura a otra (Vygotsky 1962).

Propongo una antítesis semejante sólo a título de discusión de sus implicaciones, como ilustración del tipo de evidencia "filosófica" que puede esgrimirse en las polémicas que envuelven nociones tan resbaladizas. Me 
parece que ambas posturas pueden defenderse mediante una oscura mezcla de argumentos empíricos y meramente conceptuales (entendiendo conceptual como no empírico, o al menos como no esencialmente empírico). A primera vista, el argumento esencial de Fodor para defender no ya la existencia, sino la necesidad de un lenguaje del pensamiento, es de carácter conceptual, pues rechaza la posibilidad de que podamos aprender conceptos nuevos, que suponen un sistema más rico, desde sistemas más pobres. Así, cuando defiende que el desarrollo cognoscitivo depende de estadios predeterminados de la mente, parece hacerlo por razones no empíricas (a menos que acudamos al discutible argumento de la pobreza del estímulo).

Sin embargo, no puede dudarse de que la postura de Fodor está de hecho presuponiendo la gramática generativo-transformacional como base esencial, a partir de la cual explicar el aprendizaje de un primer lenguaje como la mera formulación de hipótesis y su verificación. Claro que puede aducirse, a su vez, que tal gramática se sostiene en razones empíricas, pero es bien conocido que muchos filósofos aducen convincentes argumentos contra ella que tienen poco de empírico. Como Quine sostuvo, puede defenderse incluso que las diversas gramáticas, en la medida en que se presentan como explicaciones teóricas del lenguaje, no son sino teorías subdeterminadas por los hechos, y por tanto no dirimibles con base en ellos. Así, la mezcla de argumentos empíricos y conceptuales parece irrebasable. En tal caso, las ricas interrelaciones entre las nociones de mente y lenguaje involucrarían no sólo ciencias empíricas como la psicología, sino genuina filosofía, en este caso de la mente, del lenguaje y de la psicología.

A resultados similares puede llegarse desde el otro extremo de la antítesis. En una primera aproximación, la argumentación central de Vygotsky parte de una supuesta evidencia empírica, en el sentido de que su tesis central se formuló a partir de la observación minuciosa del desarrollo lingüístico de grupos de niños de diversas edades. Sin embargo, su noción de lenguaje interior, que no es más que una reformulación del "lenguaje egocéntrico" de Piaget, es lo suficientemente inverificable directamente como para no poder rebasar el estatus de mera construcción teórica, dependiente por ello de toda una red conceptual teórica previa. Está claro que lo mismo sucede con todos los conceptos de la ciencia, pero esa universalidad no puede esconder el hecho de que la elección de unos conceptos y no de otros permite agrupar los "datos" de múltiples maneras distintas; máxime en un caso como el que nos ocupa, en el que por manejarse nociones tan oscuras como la de "lenguaje", da la impresión de que ninguna construcción teórica posible puede servir para emitir hipótesis seriamente verificables, a menos que se reconstruya la noción en el sentido de los modernos lenguajes formales (aunque en tal caso temo que deberíamos tratar de manejar ordenadores o robots más que niños). 
Si ello es así, las razones para defender el carácter social de un primer lenguaje interiorizado, aunque conducen también a defender la identidad entre lenguaje y pensamiento, no sólo establecen un mapa completamente distinto de la mente, sino que lo hacen, en última instancia, por la mezcla de razones empíricas y conceptuales que trato de señalar. De nuevo, no es de extrañar que las ricas interacciones tradicionales entre la psicología y la filosofía (de la mente, del lenguaje y de la psicología) pervivan actualmente, y tengan todos los visos de hacerlo durante mucho tiempo. Es a partir de este tipo de consideraciones como creo que debe entrarse más directamente en el tema de las relaciones entre la filosofía del lenguaje, la filosofía de la mente y la filosofía de la psicología.

\subsection{Psicología filosófica, filosofía de la mente y filosofía de la psicología}

Una observación terminológica previa. Es ciertamente sorprendente que la expresión "psicología filosófica" esté pasada de moda, y se vea con malos ojos precisamente por muchos que hablan tranquilamente de lingüística filosófica o de lógica filosófica. Ello parece deberse al hecho de que aceptar la existencia de una parte filosófica de la psicología parece comprometer con la existencia, bien de ciertas entidades psicológicas no abordables con los métodos científicos de la psicología (sujeto, alma), bien de cierto tipo de conocimiento de ellas (empatía, comprensión, etc.) con fuertes connotaciones de filosofía tradicional; es decir, de la época en que todavía se pensaba en una especie de psicología "racional", como opuesta a la psicología científica (aunque algunos autores todavía usan la expresión sin sonrojo; véase Devitt y Sterelny 1987, cap. 15). Sin embargo, me parece una grave inconsecuencia el mantener tales renuencias cuando ello no se extiende a las expresiones hermanas relativas a la lógica y la lingüística, vista la existencia de similares peligros. No puedo, por las razones expuestas antes, más que aplaudir la expulsión de la psicología filosófica del marco de discusión actual. Lamento sólo que esa expulsión no se aplique también a las otras "disciplinas" igualmente oscurantistas.

Dicho esto podemos ya concentrarnos en el papel de la filosofía del lenguaje para la filosofía de la mente y la de la psicología. Veamos primero las relaciones entre estas últimas. La postura tradicional, que en este caso aplaudo, es la de que la filosofía de la psicología es una parte de la filosofía de la mente. Los argumentos rara vez se formulan, pero uno podría ser que la filosofía de la psicología se restringe a estudiar esa ciencia desde el punto de vista filosófico (semántico, metodológico, epistemológico, ontológico), y por tanto se ocupa de temas como la naturaleza de la representación mental, la existencia de ideas innatas (tal y como las postula cierta escuela de lingüistas) o la naturaleza de los conceptos. En cambio, la filosofía de la mente aspira además a dilucidar temas de carácter más amplio y tradicio- 
nalmente filosófico, como el de la relación entre la mente y el cuerpo, o la naturaleza de la conciencia.

Ciertamente, en ambos casos se trata de temas entre los que existe una frontera muy difusa, pero al menos parece que la aproximación a ellos debe ser diferente. En el primer caso, como filósofos, estudiamos el proceder de una ciencia en principio independiente, sus métodos y sus teorías: hacemos filosofía de la ciencia; en el segundo obramos más directamente, por más que aquella ciencia continúe siendo relevante para nuestras investigaciones, que alcanzan en este último caso un carácter más puramente conceptual. Así, propongo que las relaciones entre filosofía de la mente y filosofía de la psicología se planteen exactamente de la misma forma en que párrafos ates propuse que se hiciera con las existentes entre filosofía del lenguaje y filosofía de la lingüística.

Por las razones expuestas no puedo sino rechazar la doble propuesta de Block (1980, introducción), según la cual la filosofía de la psicología es el estudio de los temas conceptuales de la psicología (en el sentido de que tales temas son a la vez psicológicos y filosóficos), al tiempo que la filosofía de la psicología, así definida, debe tener en su seno a la filosofía de la mente (puesto que muchos de los problemas de la primera no son más que reformulaciones modernas de temas tradicionales). Me parece que la propuesta de Block es algo confusa, y esa confusión procede principalmente de su utilización de la expresión "temas conceptuales". Creo más que razonable observar que muchos de los temas que discutimos actualmente son reformulaciones más precisas e informadas científicamente de problemas tradicionales, y también que en muchos casos el planteamiento actual ha posibilitado la superación de conceptos obsoletos y distinciones conceptuales confundentes; pero de ahí a definir la filosofía de la psicología como una investigación puramente conceptual va un abismo. De no ser así, por razones similares podríamos decir que la física actual, que ha permitido la superación de la física aristotélica (un ejemplo mal usado por el propio Block), es una investigación de temas esencialmente conceptuales, pudiendo por tanto hablarse de una física "filosófica".

En cambio, creo que, aunque la ciencia permite superar viejas nociones, ello no se debe a que su investigación sea "conceptual", sino a que aplica conceptos teóricos nuevos a los materiales empíricos, de forma que tales materiales dejan por ello de ser los mismos que los que se manejaban con anterioridad. Pero al tratarse de conceptos teóricos (o de teorías, sin más; pace la nueva concepción de las teorías), dependen de todo el formato metodológico habitual de la ciencia, cosa que no hacemos al trabajar conceptualmente los problemas de la filosofía. En consecuencia, los problemas puramente conceptuales no son problemas científicos, sino filosóficos, por lo que no cabe hablar de temas conceptuales de la psicología, sino sólo de estudio filosófico (semántico, epistemológico, ontológico) de los conceptos, 
métodos y teorías científicos de la psicología. Por tales razones, es -o debe ser- la filosofía de la mente la que emprenda el trabajo puramente conceptual sobre los temas, tradicionales o no, que nos parezcan interesantes para poner orden en el entramado conceptual relevante, en parte heredado del pasado, en el que se inserta nuestra forma de hablar de la mente, sus componentes, procesos y operaciones. Debo insistir, pues, en mi propuesta de que la filosofía de la mente es el marco general en que hacemos filosofía de la psicología, y no a la inversa.

El papel de la filosofía del lenguaje es ya más fácil de establecer. Aquí las posturas de autores actuales importantes nos pueden ayudar, aunque temo que sólo a enmarcar el tema. Por escoger dos de ellas contrapuestas, tenemos que, para Searle (1983), la filosofía del lenguaje es una rama de la filosofia de la mente. El argumento es, más o menos, que puesto que el lenguaje es la expresión del pensamiento, nuestras teorías acerca de la naturaleza de la mente determinarán nuestra filosofía del lenguaje. Ahora bien, la trayectoria del propio Searle no ha sido precisamente ésa, sino incluso la contraria: sólo tras elaborar una teoría del lenguaje ha pasado a ambicionar la invasión del territorio mental. Para Dretske (en McLaughlin 1991), en cambio, aunque la conducta es secundaria y lo realmente importante es el problema de la naturaleza de la mente, no podemos entrar en ese problema sin partir de lo que la mente realmente hace, y eso está en buena medida en el estudio del lenguaje. Trataré de profundizar algo en el dilema.

Para comenzar, está fuera de duda que nuestra filosofía del lenguaje va a determinar en alguna medida nuestra concepción de la mente, al tiempo que el tipo de problemas que nos interesen de entre los que se ocupa la psicología (y por tanto su filosofía). Podría también defenderse lo contrario, pero creo que con escaso éxito, puesto que tendemos a considerar, correcta o incorrectamente, que el estudio del lenguaje es la vía regia para el estudio de la mente (del pensamiento, de la razón y otros conceptos tradicionales), y de al menos ciertas partes de la psicología (precisamente las partes que más suelen interesar al filósofo). O incluso, rebasando el enfoque puramente metodológico, tendemos a considerar que, ontológicamente hablando, ciertas propiedades lingüísticas (quizá sociales) pueden ser constitutivas de las propiedades del contenido mental mismo (del contenido de las actitudes proposicionales). Así, la prioridad disputada se manifestaría, ahora, entre la constitución del contenido mental y el significado lingüístico (véase al respecto el catálogo de posiciones que ofrecen en su introducción Loewer y Rey 1991).

En todo caso, es naturalmente innegable que siempre puede sostenerse la tesis de que ciertos filósofos, al estar digamos que irracionalmente convencidos de cierta tesis acerca de la naturaleza de la mente, "escogen" cierta concepción del lenguaje que se acomoda mejor, por sus implicaciones 
filosóficas, a su noción previa de mente, para después recorrer el camino a la inversa. Pero ya sabemos que las interpretaciones psicologistas de la filosofía son muy peligrosas y es más aconsejable atenerse a lo prima facie argumentado.

Pero hay también argumentos puramente empíricos, o si se quiere corporativos, para defender el tipo de relación que comentamos. No hay más que dar un repaso a las filosofías de la mente contemporáneas y podremos comprobar que suelen estar más que influidas por las correspondientes filosofías del lenguaje. Pensemos, por ejemplo, en Quine o en el último Wittgenstein. En tales casos, los ataques a cierta concepción del lenguaje han determinado los correspondientes rechazos de las entidades tradicionalmente asociadas a ciertas concepciones de la mente. Pensemos también en Russell y su teoría del juicio como relación múltiple. Es un simple hecho que el abandono de semejante teoría de la mente (de la creencia) se debió a argumentos genuinamente filosóficos (lógico-ontológicos) en el campo del lenguaje. O pensemos en Davidson, o en Kripke, para detectar en seguida la forma en que sus argumentos en filosofía del lenguaje han tenido implicaciones vitales para la filosofía de la mente.

Insisto; con todo ello no estoy sosteniendo la tesis a priori de que no cabe otro acceso a la mente más que el lenguaje (y por tanto su filosofía). Me limito a constatar que, para los filósofos, así suele ser. Por ello, no niego que existan otros accesos al problema de la naturaleza de la mente. En las actuales ciencias cognitivas, y su correspondiente teoría computacional o representacional de la mente (según los gustos), tenemos un modelo aparentemente alternativo, puesto que tuvo su origen en la analogía mente-ordenador. Pero, incluso así, los filósofos (que fueron quienes lo propusieron) se las han arreglado para defender el nuevo paradigma con argumentos extraídos de la filosofía del lenguaje. Es más, han sido argumentos básicamente de filosofía de lenguaje los que han hecho que muchos de tales filósofos abandonen el modelo (estoy pensando en el Putnam reciente).

Cabe, sin embargo, confundir mi posición con una tesis mucho más fuerte. Así, podría pensarse que lo que estoy defendiendo es que la filosofía del lenguaje debe, por consideraciones a priori, convertirse en la base fundamental de la filosofía (semántica, epistemología, ontología), vía cierta teoría del significado y la verdad. Sería la tesis de Dummett, que es una tesis original y ciertamente tiene sus atractivos. Pero no se trata de eso. Lo que sostengo es que los filósofos, en el uso legítimo de sus técnicas tradicionales, no suelen poder evitar servirse de los argumentos del campo de la filosofía del lenguaje para explorar conceptualmente los temas típicos de la filosofía de la mente, pero no que necesariamente deba ser así. Al fin y al cabo, la filosofía del lenguaje y la filosofía de la mente son discipli- 
nas históricamente constituidas, y, por tanto, con un precio que pagar a la tradición.

Sin embargo, no estoy tampoco diciendo que puesto que de hecho los filósofos actúan así —descripción—, deban también hacerlo — prescripción. Claro que existe un factor meramente histórico, y por tanto empírico, en la cuestión, pero no me imagino qué filosofía sería interesante hacer que no fuera de carácter esencialmente conceptual. Y si tiene ese carácter, como lo ha tenido históricamente, no puede más que estar estrechamente basada en la filosofía del lenguaje, en un sentido amplio de la expresión (no en su sentido estrechamente dummettiano de teoría del significado y la verdad).

Resumiendo: (i) existe, y debe existir, una estrecha relación entre filosofía y psicología, que procede de la relación similar entre lenguaje (conceptos) y mente (pensamiento, razón); (ii) ha de rechazarse una psicología filosófica, al igual que hicimos con una lingüística filosófica y, en el fondo, con una lógica filosófica (excepto por razones de pura imposibilidad coyuntural); (iii) la filosofía de la mente comprende a la filosofía de la psicología, y no a la inversa, lo cual se debe a su naturaleza más puramente conceptual; (iv) la filosofía del lenguaje, dada su naturaleza conceptual, es $-\mathrm{y}$ debe ser - una de las bases principales de la filosofía de la mente, al igual que al menos la de algunos de los temas más conceptuales de la filosofía de la psicología. Pasemos ya a la última de nuestras secciones, en la que, fijadas sus relaciones con disciplinas cercanas, podremos abordar de una forma más directa la naturaleza misma de la filosofía del lenguaje, una vez dentro de la filosofía misma.

\section{Filosofía del lenguaje y filosofía}

No es éste el lugar para intentar siquiera examinar las relaciones entre lenguaje y filosofía, que puede decirse constituyen lo que ha sido, en cierta forma, la historia de la filosofía toda. Por mantenernos a la vez en nuestra línea metafilosófica y dentro de lo que puede considerarse como filosofía contemporánea, en esta última sección me limitaré a abordar las relaciones entre la filosofía del lenguaje y las dos escuelas o tendencias en cuyo seno más firmemente se ha tratado de darle carta de naturaleza, tanto metodológica como metafilosófica: la filosofía lingüística y la filosofía analítica. De paso, trataré de dilucidar hasta qué punto ambas etiquetas están relacionadas entre sí y también de hacer una valoración de si merece la pena mantenerlas.

\subsection{Filosofía del lenguaje y filosofía lingüística}

Para ver hasta qué punto la expresión "filosofía lingüística" nos puede ser útil, una ojeada a la historia cercana es iluminadora. Los fundadores clá- 
sicos de lo que se entiende hoy por filosofía del lenguaje no tuvieron conciencia de que fuera necesaria una especialidad tal, ni bajo el nombre que usamos ni bajo ningún otro, con la posible excepción de la etiqueta "lógica filosófica" que introdujo Russell (véase supra). Así, creyeron simplemente que no merece la pena hacer filosofía seriamente sin un análisis previo del lenguaje empleado, en la confianza de que mediante ello se introduciría un nivel de precisión que lo hiciera manejable, al tiempo que se desvelarían muchos de los presupuestos que dificultaban el progreso hacia sus respectivos objetivos. Sin embargo, no hicieron de ello programa filosófico alguno. Fueron los miembros del Círculo de Viena quienes, incluso a título de manifiesto o programa filosófico, hicieron explícita la idea de que la solución de los problemas filosóficos requiere su reformulación dentro de una concepción específica del lenguaje, sin duda heredera del "lenguaje ideal" más o menos implícito en muchos de los análisis de Frege, Russell y Wittgenstein.

Ahora bien, tal reformulación habría de suponer no sólo un trabajo previo de desbrozamiento del confuso terreno lingüístico por recorrer, sino una auténtica pavimentación, realizada con la vista puesta en los métodos de la lógica y la matemática. En esa línea, los neopositivistas llegaron a defender que, a fin de cuentas, los problemas filosóficos surgen sólo porque no se es consciente de la verdadera naturaleza del lenguaje, que no esconde ya un conjunto de formas lógicas aislables por separado, sino toda una sintaxis lógica, cuya pérdida de vista conduce a las mayores confusiones. Se trataba, pues, de reconstruir sistemáticamente un lenguaje que pusiera tal sintaxis de manifiesto, hasta disolver completamente los problemas filosóficos tradicionales, al tiempo que se mostraba la natureza "lingüística" de las ciencias formales. No vamos a recorrer la historia en detalle, pero hay al menos que decir que la imposibilidad de superar las implicaciones limitativas de los resultados de Gödel llevaron al convencimiento de que no era posible hallar ninguna "otra completud" para la sintaxis lógica, al tiempo que los de Tarski impulsaban hacia la semántica, donde, como es sabido, los sueños iniciales dieron al traste. Así, el viejo proyecto del lenguaje ideal terminaba sus días.

Sin embargo, la antorcha fue recogida, más que sorprendentemente, por otra escuela, también heredera de Wittgenstein, y no necesariamente del último; para ello no hay más que recordar que el tipo de filosofía característica de esta escuela se practicaba ya en parte durante los años treinta (Ryle 1932). Los llamados filósofos del lenguaje ordinario (o escuela de Oxford) llegaron a la constatación de que, aunque es cierto que los problemas filosóficos surgen por descuido en nuestro tratamiento del lenguaje, el método que debe seguirse no es reconstruir éste, sino simplemente "dejarlo como está", con la única precaución de respetar la profunda relación existente entre significado y uso. Curiosamente, como fue perfectamente constatado 
por muchos en los años sesenta (por ejemplo, Rorty 1967, introducción; véase también Rorty 1990), semejante proceder tenía lo esencial en común con el positivismo lógico: (i) pensar que la solución de los problemas filosóficos es "lingüística", y (ii) creer que existen criterios que podemos seguir para tomar como modelo una cierta concepción del lenguaje, concepción que sería la "ideal" para llevar adelante el programa (aunque no se tratara aquí de reconstrucción alguna, sino más bien de una descripción cuidadosa de los mecanismos hasta entonces ocultos, muchos de ellos de carácter pragmático, como creyó Austin).

Pues bien, es entonces, a finales de los años cincuenta y principios de los sesenta, cuando el uso de la expresión "filosofía lingüística" se comienza a generalizar, siempre para referirse tanto a la filosofía del positivismo lógico y sus herederas como a la del lenguaje ordinario, dado que tanto la una como la otra compartían los presupuestos de carácter lingüístico descritos, que eran tanto metodológicos como sustantivos, pues en filosofía la defensa de un método ha de basarse en criterios filosóficos previos. Ahora bien, puesto que por los años sesenta era sólo la segunda de esas escuelas la que estaba viva (aunque ciertamente ya atacada de grave enfermedad), la expresión de marras solía aplicarse más bien para referirse exclusivamente a ella, sobre todo porque los nuevos teóricos de la forma lógica (Quine, Davidson) no habían desarrollado todavía sus nuevas teorías, que en parte pretendían mantener la antorcha del positivismo lógico, aunque ya dentro del nuevo paradigma holístico.

Así, se pasó a describir la filosofía del lenguaje ordinario (o de Oxford) con la etiqueta "filosofía lingüística", entendiéndose ésta fundamentalmente como un método, más o menos lexicográfico, basado en el estudio de casos concretos y presidido por el recurso básico al argumento del "paradigm case", o inferencia del uso real de las palabras, para la resolución (o disolución) de los problemas filosóficos. Una ilustración, y un ataque feroz contra ese tipo de filosofía, fue Gellner 1959, que es muy útil para comprender la gran implantación de semejante filosofía en el Reino Unido, así como el gran rechazo que suscitaba en quienes, como Russell, dieron en pensar que se trataba nada más que de la renuncia al trabajo duro (de una "filosofía sin lágrimas").

También en Estados Unidos adoptaron la etiqueta, aunque algo más tarde, pero sin demasiada claridad en cuanto a su extensión o campo de aplicación. Por ejemplo, Katz $(1966,1971)$ la usaba para referirse tanto a la línea reconstructiva como a la de Oxford (en la línea de Rorty 1967), mientras que Searle (1969) se inclinaba por el uso más restrictivo, entendiendo que filosofía lingüística es el nombre de un método, presumiblemente el de los filósofos del lenguaje ordinario. De cualquier manera, ambos casos son ilustrativos de cómo iba evolucionando la filosofía del lenguaje. Para Katz, era necesario superar las severas limitaciones de la filosofía lingüísti- 
ca mediante el estudio sistemático de las implicaciones del nuevo paradigma generativo-transformacional. Así, la gramática chomskyana aportaba el marco en el cual la filosofía lingüística habría de desarrollarse, siempre en la misma línea de una búsqueda de la solución de problemas filosóficos a partir de una concepción -en este caso una supuesta teoría científicadel lenguaje. En cambio, para Searle, el modelo que había que seguir era el ya creado por Austin, una vez sistematizado, y con la importante novedad de convertirse no ya en un mero "método" filosófico, sino en toda una "filosofía del lenguaje". Me parece que con esa contraposición fallece, por consunción, la expresión "filosofía lingüística". No creo que valga la pena seguir usándola, salvo en su sentido histórico, es decir, asociada a cierto periodo caracterizado por el clímax de lo que desde entonces se conoce como "el giro lingüístico".

Resumiendo: la expresión "filosofía lingüística" no tiene más relación con lo que hoy entendemos por filosofía del lenguaje que la puramente histórica, según la cual, en los años cincuenta y sesenta, se llamó filósofos lingüísticos preferentemente a los filósofos del lenguaje ordinario, y a veces, a falta de otra expresión mejor, a los practicantes del tipo de filosofía del empirismo lógico. La filosofía del lenguaje como la entendemos hoy es algo mucho más amplio, pues incluye ideas y métodos que han ensanchado considerablemente el panorama en que la expresión que estudiamos se originó, y que se acercan más a lo que a veces se entiende por filosofía analítica. Por tanto, no puedo aceptar que "filosofía lingüística" se utilice como expresión más o menos sinónima de "filosofia analítica" (Acero 1985), que tuvo y tiene un contenido distinto, y sobre la que ahora diré también algo.

\subsection{Filosofía del lenguaje y filosofía analítica}

Dilucidar el sentido de la expresión "filosofía analítica" es mucho más difícil que hacerlo con el de "filosofía lingüística". Dos son las líneas posibles: la histórica y la sistemática. Históricamente, por filosofía analítica se ha entendido meramente uno de los tres bloques filosóficos de que habló juiciosamente Ferrater Mora (1969), caracterizado por ser el practicado en los países anglosajones (con el importante añadido de la herencia alemana y austriaca procedente de la huida del nazismo), siendo los otros dos la filosofía "continental", inspirada sobre todo en los filósofos alemanes del neohegelianismo y neokantismo (incluyendo a Husserl y el existencialismo francés), y la filosofía "marxista".

Sistemáticamente, la cosa es mucho menos clara. No creo que se pueda hacer mucho más que tratar de caracterizar la filosofía analítica por lo que a veces se llama método del análisis filosófico (Ferrater 1974, Acero 1985). Lo malo es que entonces no podemos más que señalar también un recorrido histórico concretado en una lista de autores, a menos que debili- 
temos tanto la caracterización que nos limitemos a mencionar generalidades como: esfuerzo por construir, o aceptar, cierta concepción del lenguaje como prolegómeno al trabajo filosófico; esfuerzo por la claridad y la precisión; cierto conocimiento y respeto por los filósofos fundadores (Frege, Russell, Wittgenstein); cierto grado de inspiración en la lógica matemática; cierto conocimiento y respeto por los resultados de la ciencia y su filosofía; la consiguiente moderación en las tesis metafísicas; cierto seguimiento - e inspiración en ellos- de los grandes filósofos herederos de los fundadores (Quine, Putnam), etc. Claro que, entonces, más que de un método hay que hablar meramente de una línea o tradición.

No obstante, tanto en el sentido histórico como en el sistemático, creo que puede defenderse que la filosofía analítica, o al menos la tradición analítica, sigue existiendo. Como constatación de hecho no hay más que observar la forma, más o menos estricta, tanto en Estados Unidos como en el resto de América y en Europa, en que los filósofos pueden clasificarse todavía como pertenecientes o no a la tradición analítica, cuyas obras suelen salir a la luz en las mismas revistas, editoriales, congresos, etc. Hay otros factores que han venido a complicar el panorama, pero me parece que son fácilmente aislables. El principal de ellos es la aparición de un nuevo paradigma, el de las "ciencias cognitivas", en el que participa la mayoría de los filósofos actuales de tradición analítica, fundamentalmente por tratarse de un marco lo suficientemente fértil y bien estructurado como para facilitar las líneas tradicionales de trabajo con nuevos instrumentos, siendo el principal de ellos la teoría computacional-representacional de la mente, favorecedora de un enfoque lingüístico de muchos problemas filosóficos.

Dentro de esa concepción de la filosofía analítica hemos de insertar lo que hoy se entiende por filosofía del lenguaje, o al menos lo que yo entiendo que cae -o debería caer - bajo tal denominación. En ese sentido, la filosofía del lenguaje sigue siendo de importancia vital dentro de la filosofía de tradición analítica como un todo, tanto porque ha de considerarse como una especie de materia de formación básica que da un acceso fructífero a otras materias, como porque el tipo de problemas que maneja constituye, metodológicamente hablando, un desbrozamiento útil del terreno a la hora de aspirar a hallar soluciones a esos problemas. Así entendida, y sin hablar de contenidos concretos, creo que la filosofía del lenguaje continúa - y debería continuar - siendo fundamento de la filosofía de tradición analítica. Sin embargo, como expliqué antes, ello no debe entenderse en el sentido fuerte de Dummett, que presupone que es — sólo— la teoría del significado, entendida de cierta manera, la que constituye - $\mathrm{y}$ debe constituir- la base de esa filosofía del lenguaje. 


\section{BIBLIOGRAFÍA}

Acero, J.J., 1985, Filosofía y análisis del lenguaje, Cincel, Madrid.

Acero, J.J., E. Buston y D. Quesada, 1982, Introducción a la filosofía del lenguaje, Cátedra, Madrid.

Barwise, J. (comp.), 1977, Handbook of Mathematical Logic, Horth-Holland, Amsterdam.

Block, N. (comp.), 1980, Readings in Philosophy of Psychology, Harvard University Press, Cambridge, Mass., vol. 1.

Danto, A.C., 1968, What Philosophy Is: A Guide to the Elements, Harper Torchbooks, Nueva York. [Versión en castellano: Qué es filosofía, trad. N. Míguez, Alianza, Madrid, 1976.]

Devitt, M. y K. Sterelny, 1987, Language and Reality, Basil Blackwell, Oxford.

Dretske, F., 1991, “Dretske's Replies: Knowledge: Sanford and Cohen”, en McLaughlin 1991, cap. 10, pp. 185-196.

Dummett, M., 1978, Truth and Other Enigmas, Duckworth, Londres. [Versión en castellano: La verdad y otros enigmas, trad. Alfredo Herrera, Fondo de Cultura Económica, México, 1990.]

— 1973, Frege. The Philosophy of Language, Duckworth, Londres.

Ferrater Mora, J., 1974, Cambio de marcha en filosofía, Alianza, Madrid.

—_, 1969, La filosofía actual, Alianza, Madrid.

Fodor, J., 1975, The Language of Thought, Thomas Y. Crowell, Nueva York. [Versión en castellano: El lenguaje del pensamiento, trad. Jesús Fernández Zuliaca, Alianza, Madrid, 1984.]

Gabbay, D. y F. Guenthner (comps.), 1983-1989, Handbook of Philosophical Logic, Reidel, Dordrecht, vols. I-IV.

Gamut, L.T.F., 1991, Logic, Language and Meaning, The University of Chicago Press, Chicago, 2 vols.

Garrido, M. (comp.), 1989, Lógica y lenguaje, Tecnos, Madrid.

Gellner, E., 1959, Words and Things, Victor Gollanz, Londres (2a. ed., 1979). [Palabras y cosas, trad. Mónica Acherdoff, Tecnos, Madrid, 1962.]

Gödel, K., 1994, Ensayos inéditos, ed. F. Rodríguez Consuegra, Grijalbo-Mondadori, Barcelona.

Katz, J.J., 1971, The Underlying Reality of Language and Its Philosophical Import, Harper and Row, Nueva York (también como Linguistics and Philosophy, Allen and Unwin, Londres). [Versión en castellano: La realidad subyacente del lenguaje y su valor filosófico, trad. Conxita Lleó, Alianza, Madrid, 1975.]

— , 1966, The Philosophy of Language, Harper and Row, Nueva York. [Versión en castellano (muy deficiente): Filosofía del lenguaje, Martínez Roca, Barcelona, 1971.]

Loewer, B. y G. Rey (comps.), 1991, Meaning in Mind. Fodor and his Critics, Basil Blackwell, Oxford.

Lycan, W., 1984, Logical Form in Natural Language, Bradford Books, Cambridge, Mass.

McLaughlin, B. (comp.), 1991, Dretske and his Critics, Basil Blackwell, Oxford.

Millikan, R.G., 1984, Language, Thought and Other Biological Categories, Bradford/The MIT Press, Cambridge, Mass. 
Montague, R., 1974, Formal Philosophy, ed. R.H. Thomason, Yale University Press, New Haven. [Versión parcial en castellano: Ensayos de filosofía formal, trad. D. Quesada, Alianza, Madrid, 1977.]

Otero, C.P., 1989, "Filosofía del lenguaje", en Garrido 1989, pp. 195-255.

_-, 1984, La revolución de Chomsky, Tecnos, Madrid.

Quine, W.V.O., 2001, Acerca del conocimiento científico y otros dogmas, ed. F. Rodríguez Consuegra, Paidós, Barcelona, 2001.

_-, 1969, Ontological Relativity and Other Essays, Columbia University Press, Nueva York. [Versión en castellano: La relatividad ontológica y otros ensayos, trad. M. Garrido y J.Ll. Blasco, Tecnos, Madrid, 1974.]

—_, 1960, Word and Object, The MIT Press, Cambridge, Mass. [Versión en castellano: Palabra y objeto, trad. M. Sacristán, Labor, Madrid, 1968.]

Rodríguez Consuegra, F., 2002, Estudios de filosofía del lenguaje, Comares, Granada.

__, 1992, "La reducción ontológica y sus problemas", Crítica, vol. XXIV, no. 70, pp. 17-64.

—_, 1991, "Números, objetos y estructuras", Crítica, vol. XXIII, no. 68, pp. 7-86.

Rorty, R., 1990, El giro lingüístico, versión en castellano de la introducción a 1967

(más dos puestas al día posteriores), trad. Gabriel Bello, Paidós/Universidad Autónoma de Barcelona/Instituto de Ciencias de la Educación, Barcelona.

- (comp.), 1967, The Linguistic Turn, The Chicago University Press, Chicago.

Russell, B., 1999, Análisis filosófico, ed. F. Rodríguez Consuegra, Paidós/Universidad Autónoma de Barcelona/Instituto de Ciencias de la Educación, Barcelona.

_ 1973 , Essays in Analysis, ed. D. Lackey, Allen and Unwin, Londres.

—_, 1951, "Is Mathematics Purely Linguistic?", en 1973, pp. 295-306. [Versión en castellano: “iEs la matemática puramente lingüística?”, en Russell 1999, pp. 113-127.]

_-, 1919, Introduction to Mathematical Philosophy, Allen and Unwin, Londres.

_-, 1914, Our Knowledge of External World, Allen and Unwin, Londres.

- (con A.N. Whitehead), 1910, Principia Mathematica, Cambridge University Press, Londres, vol. I. Los vols. II y III, en 1912 y 1913, respectivamente (2a. ed., con nueva introducción (de Russell solo), 1927).

Ryle, G., 1932, "Systematically Misleading Expressions", Proceedings of the Aristotelian Society, vol. 32, pp. 139-170.

Sainsbury, R.M., 1991, Logical Forms, Basil Blackwell, Oxford.

Schiffer, S., 1987, Remnants of Meaning, The MIT Press, Cambridge, Mass.

Searle, J., 1983, Intentionality: An Essay in the Philosophy of Mind, Cambridge University Press, Cambridge.

— 1969, Speech Acts: An Essay in the Philosophy of Language, Cambridge University Press, Londres. [Versión en castellano: Actos de habla: ensayo de filosofía del lenguaje, trad. Luis M. Valdés Villanueva, Cátedra, Madrid, 1980.]

Valdés, L.M. (comp.), 1991, La búsqueda del significado, Tecnos/Universidad de Murcia, Madrid.

Vygotsky, L.S., 1962, Pensamiento y lenguaje, La Pléyade, Buenos Aires (1a. ed.: 1934). 